\title{
The impact of hydrogenation conditions on the temperature and strain discrimination of type I and type IA Bragg grating sensors
}

Kyriacos Kalli, A. George Simpson, Kaimin Zhou, Lin Zhang, lan Bennion

Kyriacos Kalli, A. George Simpson, Kaimin Zhou, Lin Zhang, lan Bennion, "The impact of hydrogenation conditions on the temperature and strain discrimination of type I and type IA Bragg grating sensors," Proc. SPIE 6189, Optical Sensing II, 61891T (22 April 2006); doi: 10.1117/12.662311 


\title{
The impact of hydrogenation conditions on the temperature and strain discrimination of Type I and Type IA Bragg grating sensors
}

\author{
Kyriacos Kalli ${ }^{1 *}$, A George Simpson ${ }^{2}$, Kaimin Zhou ${ }^{2}$, Lin Zhang ${ }^{2}$ and Ian Bennion ${ }^{2}$ \\ ${ }^{1}$ Higher Technical Institute, C. Kavafi Street, Nicosia, 2152 Cyprus \\ 2 Photonics Research Group, Aston University, Birmingham B4 7ET United Kingdom
}

\begin{abstract}
We report experimental findings for tailoring the temperature and strain coefficients of Type I and Type IA fibre Bragg gratings by influencing the photosensitivity presensitisation of the host optical fibre. It is shown that by controlling the level of hydrogen saturation, via hot and cold hydrogenation, it is possible to produce gratings with lower thermal coefficients. Furthermore, there is a larger difference between the Type I and Type IA thermal coefficients and a significant improvement in the matrix condition number, which impacts the ability to recover accurate temperature and strain data using the Type1-1A dual grating sensor.
\end{abstract}

Keywords: Optical fibre sensors, fibre Bragg gratings, Type IA grating, photosensitivity, temperature and strain sensors

\section{INTRODUCTION}

Type IA fibre Bragg gratings have attracted interest within the sensor community based on their unique spectral and physical characteristics. They are readily identified by their spectral attribute of a large red shift in the Bragg wavelength $\left(\lambda_{\mathrm{B}}\right)$ of the grating during inscription that accompanies an increase in the mean core index ${ }^{1}$. It is recognised that this red shift is dependent on fibre type and hydrogenation conditions, and for a highly doped fibre, such as B/Ge codoped fibre, is typically $15-20 \mathrm{~nm}$, whereas the wavelength shift for SMF28 is lower at $5-8 \mathrm{~nm}^{2}$. The maximum wavelength shift translates to an increase in the mean index of up to $2 \times 10^{-2}$. We have previously shown a strong correlation between the growth of the $\mathrm{OH}$ absorption band formation in the optical fibre during prolonged $\mathrm{UV}$ exposure and the increase in the mean index change of the fibre grating. This change results from the hydrogen combining with $\mathrm{Si}$ and $\mathrm{Ge}$ centres in the fibre to form stable $\mathrm{SiOH}$ and $\mathrm{GeOH}$ groups, the latter of which has the greater impact on the strength and peak location of the $1400 \mathrm{~nm}$ absorption band. As a result of this fundamental material modification Type I and Type IA gratings have been written in the same fibre with a common phase mask, yet with central reflecting peaks more than $14 \mathrm{~nm}$ apart after annealing. More importantly, this change in the mean index of the fibre core results in their key physical attribute, that they exhibit the lowest temperature coefficient of all grating types reported to date; this makes them attractive for use in a temperature compensating, dual grating sensor ${ }^{3}$.

Here we focus on the effect of hydrogenation on the thermal coefficients of Type I and Type IA grating sensors and how this affects the recovery of accurate temperature and strain data. We control the degree of photosensitivity presensitisation of the host optical fibre by controlling the level of hydrogen saturation, via hot and cold hydrogenation. We will show that it is possible to produce Type IA gratings with low thermal coefficients, particularly when compared to Type I gratings. Furthermore, tailoring a large difference between the Type I and Type IA thermal coefficients leads to a significant improvement in the matrix condition number, this impacts the ability to recover accurate temperature and strain data when using a Type1-1A dual grating sensor. We will show that the improvement is significant and makes this dual grating scheme well suited to dual measurand applications, performing well compared with other, more elaborate techniques schemes that utilize multiple Bragg gratings to simultaneously decouple temperature and strain.

\section{HYDROGENATION CONDITIONS AND GROWTH CURVES}

It is well documented that optical fibres hydrogenated at lower temperatures achieve a higher hydrogen concentration within the core but require significantly longer saturation times ${ }^{4}$. The concentration of hydrogen molecules and the rate at which these molecules diffuse into the core of the optical fibre depend on the temperature and pressure at which the

Optical Sensing II, edited by Brian Culshaw, Anna G. Mignani, Hartmut Bartelt, Leszek R. Jaroszewicz, Proc. of SPIE Vol. 6189, 61891T, (2006) · 0277-786X/06/\$15 · doi: 10.1117/12.662311 
fibre is soaked. The concentration of hydrogen molecules in the optical fibre core at saturation (the equilibrium solubility), $\kappa_{\text {sat }}$, is given by:

$$
\left.\kappa_{\text {sat }}=3.3481 p \exp \left[\frac{8670 \mathrm{~J} / \mathrm{mol}}{R T}\right][\mathrm{ppm}]\left(1 \mathrm{ppm}=10^{-6} \text { moles } \mathrm{H}_{2} / \mathrm{mole}^{\mathrm{SiO}}\right)_{2}\right)
$$

where $p$ is the pressure of the hydrogen in atmospheres, $T$ is the temperature in Kelvin, and $R$ is the gas constant $\left(8.31451 \mathrm{JK}^{-1} \mathrm{~mol}^{-1}\right)$. The saturated hydrogen concentration increases linearly with pressure and decreases as the temperature increases. The variance in $\kappa_{\text {sat }}$ for changes of $p$ and $T$ is shown in figure 1 (a). The diffusivity of hydrogen molecules in silica is given by ${ }^{4}$ :

$$
d_{H_{2}}=2.83 * 10^{-4} p \exp \left[\frac{-40190 \mathrm{~J} / \mathrm{mol}}{R T}\right]\left[\mathrm{cm}^{2} \mathrm{~s}^{-1}\right]
$$

and increases with both pressure and temperature.

The variance in $d_{H_{2}}$ for changes of $p$ and $T$ is shown in figure 1 (b). Figure 1 shows the trade off that must be made when hydrogenating optical fibres; higher temperatures mean that it is possible to hydrogenate fibres relatively quickly, but only at the expense of the final concentration of hydrogen in the core of the fibre.
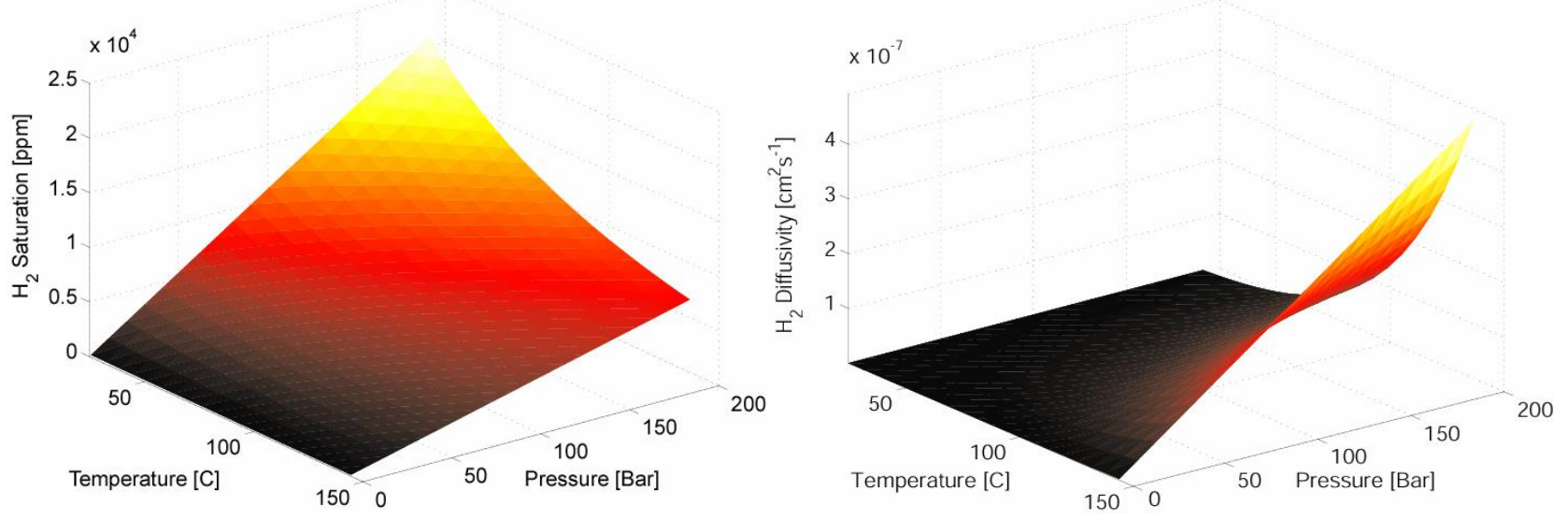

Figure 1. (a) (left) The variance in the hydrogen saturation levels of a silica fibre core for changes of $\mathrm{p}$ and $\mathrm{T}$, and (b) (right) The variance in the diffusivity of hydrogen in silica for changes of $\mathrm{p}$ and $\mathrm{T}$.

The solution on the axis for outward diffusion in cylindrical geometry is conveniently given by $\mathrm{Crank}^{5}$ as:

$$
\frac{C}{\kappa_{\text {sat }}}=1-\exp \left(-\frac{a^{2}}{4 d_{H_{2}} t}\right)
$$

where $C$ is the concentration of hydrogen in the fibre and $a$ is the fibre radius. 
In order to determine how the type of hydrogenation affects the formation of fibre Bragg gratings, we prepared two identical batches of fibre into which were inscribed Type I and Type IA gratings. Each batch consisted of Corning SMF28 standard telecommunications fibre and Verillion B/Ge co-doped fibre. One batch was hydrogenated at $80^{\circ} \mathrm{C}, 190 \mathrm{Bar}$ for 93 hours and cooled to room temperature over 24 hours by which time the pressure was 160Bar; the other batch was hydrogenated in excess of four months at 180Bar and at room temperature. Both samples were hydrogenated for times well in excess of the equilibrium time and we calculated the hydrogen concentration within the fibre samples using equation 1 based on the hydrogenation conditions outlined above. These results are shown in Table 1.

Table 1. A summary of the hydrogenation conditions for the hot and cold hydrogenated samples, showing the time, temperature and pressure of hydrogenation and the calculated saturation level within the fibre core

\begin{tabular}{c|ccc|c}
\hline & Temperature (K) & Pressure (Atmosphere) & Time (Hours) & $\boldsymbol{\kappa}_{\text {sat }}(\mathbf{p p m})$ \\
Hot & 353 & 178 & 93 & 11400 \\
Cold & 298 & 188 & $>384$ & 21000 \\
\hline
\end{tabular}

Gratings were inscribed using the scanning phase mask technique illuminated with a cw UV laser source operating at $244 \mathrm{~nm}$. A $1-\mathrm{mm}$ aperture ensured an accurate top-hat exposure profile along the length of the grating ${ }^{3}$. The stage was scanned at $1 \mathrm{mms}^{-1}$ with $130 \mathrm{~mW}$ optical power delivered to the fibre. Figure 2 shows the growth rate for the hot and cold samples in the different fibre types. There is a clear trend showing that the fibres hydrogenated at room temperature grow faster then those heated during the hydrogenation process.
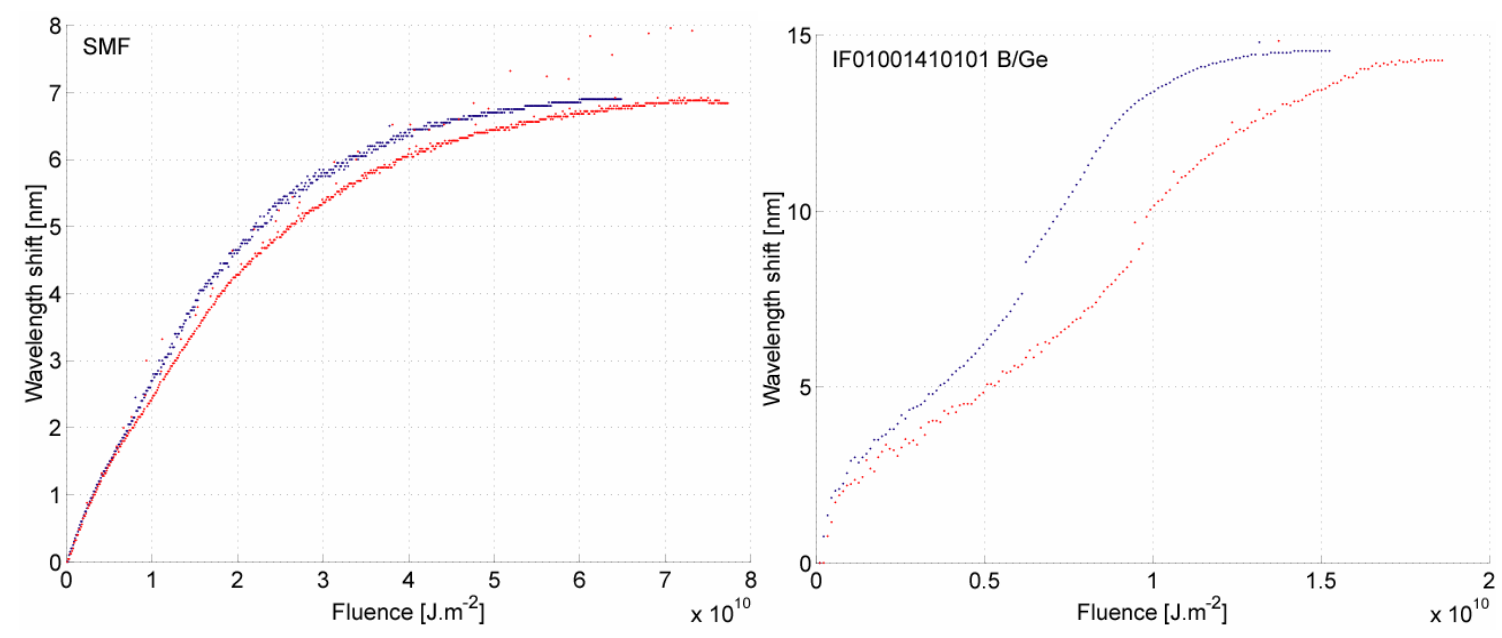

Figure 2. Growth rate of Type IA gratings in the indicated fibre types, for hot (grey) and cold (black) hydrogenation conditions.

In order that the Type IA gratings would have a reference grating whose properties are well understood, a 1-mm Type I grating was written within the same section of each fibre. Figure 3 shows the spectra of each sample before (upper) and after (lower) annealing at $80^{\circ} \mathrm{C}$ for 96 hours, and highlights a number of differences between the fabricated gratings; notable examples are the gratings written in Verillion IF01001410101 B/Ge co-doped fibre, where both the IA and I gratings differ significantly in amplitude and the Type I grating would seem to be slightly offset in wavelength. The gratings in SMF-28 are comparable in hot and cold hydrogenated samples and do not exhibit any significant spectral characteristics other than the anticipated short wavelength losses associated with the hydrogenation. The wavelength difference between Bragg resonances of the Type I and IA gratings is summarised in Table 2 for the pre- and postannealed gratings. 

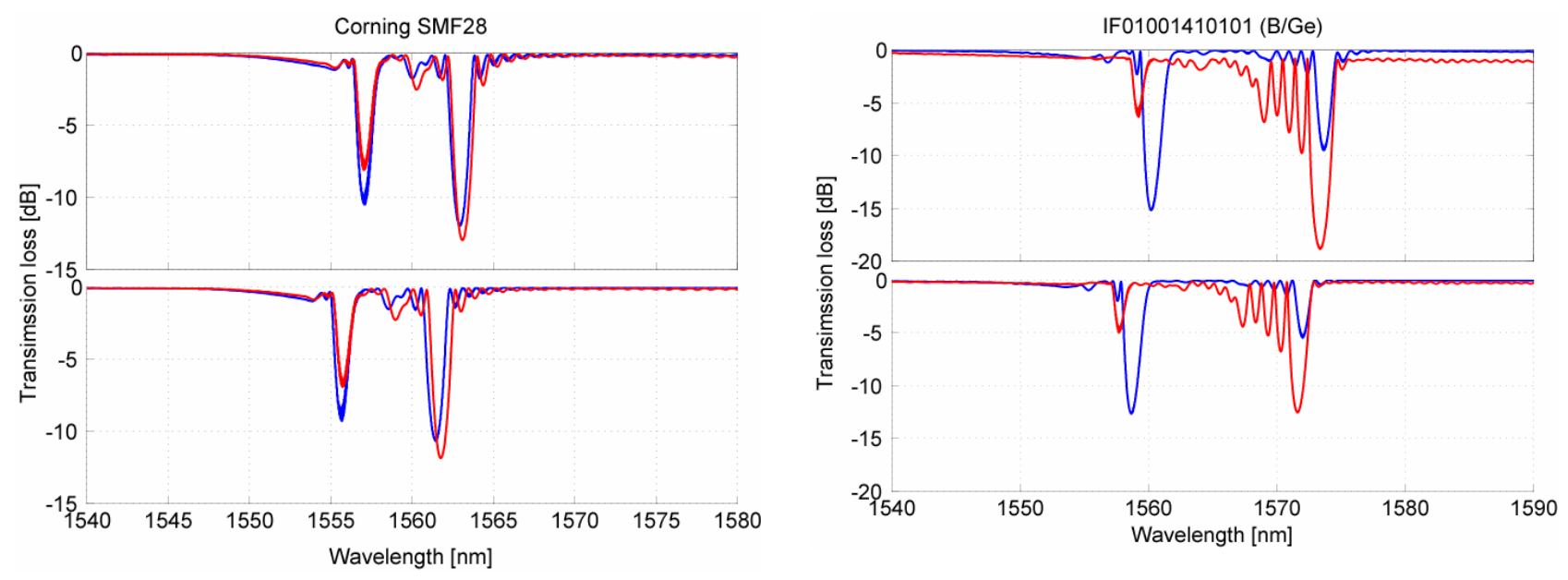

Figure 3. Pre- (upper traces) and post- (lower traces) annealing spectra for Type I and Type IA gratings written in various fibre types (as indicated) for hot (grey) and cold (black) hydrogenation conditions

\section{TEMPERATURE AND STRAIN COEFFICIENTS}

The thermal and strain coefficients of the gratings were measured by placing individual gratings on a temperature controlled block within an insulated chamber and mounted on translation stages. The temperature was controlled by means of a Peltier device connected to a standard, computer operated temperature controller. Temperature feedback was made possible by the placement of a calibrated thermistor and the Bragg wavelength was measured by passing broadband IR radiation from a powerful ASE source through the fibre to an OSA with 0.06nm resolution. The centroidfitting algorithm (CFA) was used to locate the Bragg wavelength peak recorded by the OSA. A computer was used to set and record the temperature of the grating and the OSA traces; plotted in Figure 3 for each grating and fibre type, as indicated. The thermal coefficients are summarised in Table 2. The values we have measured may be compared with independent studies on Type I and IA grating temperature coefficients (although no strain responsivities are quoted) ${ }^{6}$.

Table 2. A summary of the data highlighting the differences between hot and cold hydrogenation in the different fibre types.

\begin{tabular}{c:ccccccc}
\hline Fibre type & $\begin{array}{c}\text { Hydrogenation } \\
\text { conditions }\end{array}$ & Manufacturer & $\lambda_{B R}^{I}(T)$ & $\lambda_{B R}^{I A}(T)$ & $\frac{\lambda_{B R}^{I}(T)}{\lambda_{B R}^{I A}(T)}$ & $\lambda_{B R}^{I A}-\lambda_{B R}^{I}$ & $\begin{array}{c}\lambda_{B R}^{I A}-\lambda_{B R}^{I} \\
\text { annealed }\end{array}$ \\
SMF28 & hot & Corning & 10.54 & 10.427 & $\mathbf{1 . 0 8 4}$ & 5.848 & $\mathbf{5 . 7 6 1}$ \\
$\mathrm{SMF} 28$ & cold & Corning & 10.28 & 9.958 & $\mathbf{3 . 2 3 3}$ & 6.076 & $\mathbf{6 . 0 3 2}$ \\
$\mathrm{B} / \mathrm{Ge}$ & hot & Verillion & 9.146 & 8.27 & $\mathbf{1 0 . 5 9 2}$ & 14.147 & $\mathbf{1 3 . 9 2 5}$ \\
$\mathrm{B} / \mathrm{Ge}$ & cold & Verillion & 8.531 & 7.403 & $\mathbf{1 5 . 2 3 7}$ & 13.474 & $\mathbf{1 3 . 3 7 5}$ \\
\hline
\end{tabular}

Figure 4 shows that both the hot and cold hydrogenated fibres display an unambiguous trend, the cold samples possess a lower temperature coefficient $\left(\lambda_{B R}(T)\right)$. Moreover the difference between the $\lambda_{B R}(T)$ values for Type I and IA gratings is larger when the samples are hydrogenated at $25^{\circ} \mathrm{C}$ in preference to $80^{\circ} \mathrm{C}$. It is unclear from this experiment alone whether the effect is a result of an increased hydrogen level caused by the longer term, lower temperature hydrogenation, but we believe that this is a reasonable assumption. Regardless, this is almost certainly related to the presence of increased $\mathrm{GeOH}$ centres that are formed during the UV treatment of the fibre. The wavelength to strain responsivity displays a smaller but measurable difference that results primarily from differences in the initial centre wavelengths rather than any variation in the material properties. 

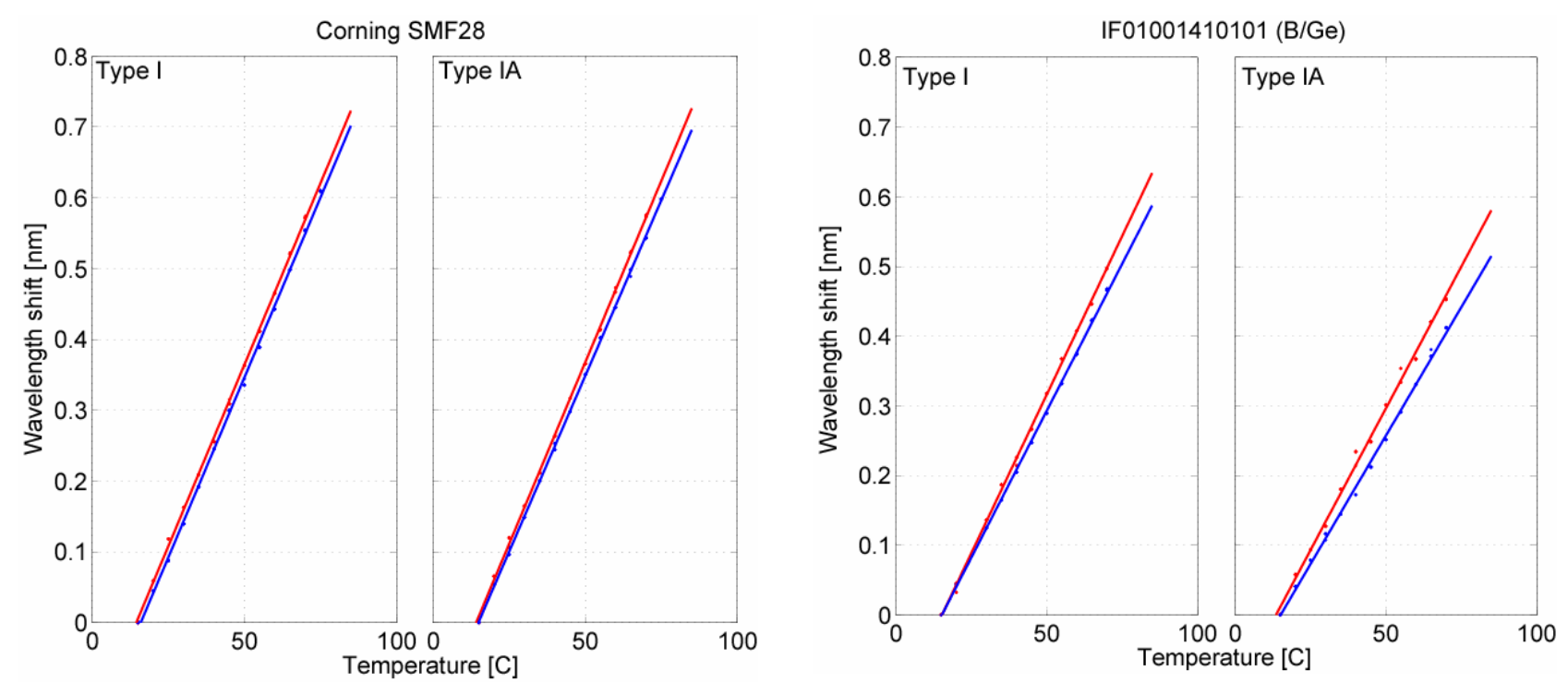

Figure 4. Plots showing the thermal coefficients for Type I (left-hand traces) and Type IA (right-hand traces) gratings written in various fibre types (as indicated) for hot (grey) and cold (black) hydrogenation conditions.

We can compare errors in the derived measurements with those of the ideal case. Ideally, the errors in strain $(\delta \varepsilon)$ and temperature $(\delta T)$ are given by ${ }^{7}$ :

$$
\left(\begin{array}{c}
\delta T \\
\delta \varepsilon
\end{array}\right)=\left(\begin{array}{c}
\delta \phi_{1} / K_{1 T} \\
\delta \phi_{2} / K_{2 \varepsilon}
\end{array}\right)
$$

where $\delta \phi_{1}$ and $\delta \phi_{2}$ are the measurement errors of parameters $\phi_{1}$ and $\phi_{2}$, in this case the wavelength. The error increases for strain and temperature according to ${ }^{8}$ :

$$
|\delta \varepsilon|=\frac{\left|K_{2 T}\right|\left|\delta \phi_{1}\right|+\left|K_{1 T}\right|\left|\delta \phi_{2}\right|}{\left|K_{1 T} K_{2 \varepsilon}-K_{2 T} K_{1 \varepsilon}\right|}
$$

and,

$$
|\delta T|=\frac{\left|K_{2 \varepsilon}\right|\left|\delta \phi_{1}\right|+\left|K_{1 \varepsilon}\right|\left|\delta \phi_{2}\right|}{\left|K_{2 T} K_{1 \varepsilon}-K_{1 T} K_{2 \varepsilon}\right|}
$$

An alternative description has been provided in ${ }^{9}$ where the errors in $\phi_{1}$ and $\phi_{l}$ have been converted to an error ellipse in the $(\varepsilon, T)$ plane. 
For the case of the Verillion $\mathrm{B} / \mathrm{Ge}$ fibre the strain coefficients for the Type I and Type IA gratings are $\lambda^{I}{ }_{B R}(\varepsilon)=$ $0.818 \mathrm{pm} / \mu \varepsilon$ and $\lambda^{I A}{ }_{B R}(\varepsilon)=0.828 \mathrm{pm} / \mu \varepsilon$, respectively. The strain and temperature errors associated with their respective coefficients are $\pm 15.3 \mu \varepsilon / \mathrm{pm}$ and $\pm 1.44^{\circ} \mathrm{C} / \mathrm{pm}$, for the hot hydrogenation in Verillion $\mathrm{B} / \mathrm{Ge}$ fibre and $\pm 11.2 \mu \varepsilon / \mathrm{pm}$ and $\pm 1.15^{\circ} \mathrm{C} / \mathrm{pm}$ for the cold hydrogen loading in the same fibre type. These values compare with errors of $\pm 12 \mu \varepsilon / \mathrm{pm}$ and $\pm 1.3^{\circ} \mathrm{C} / \mathrm{pm}$ measured by Xu et al. ${ }^{10}$ for two superimposed gratings at markedly different wavelengths. Therefore the approach of controlling the degree of hydrogenation to affect the temperature coefficients of a photosensitive fibre can prove to be very useful for a dual grating sensor used to differentiate between strain and temperature. Furthermore, a useful mathematical tool for quickly assessing the accuracy of a dual sensor arrangement is to calculate the condition number of the sensor matrix ${ }^{11}$. The condition number of a matrix measures the sensitivity of the solution of a system of linear equations to errors in the data. It gives an indication of the accuracy of the results from matrix inversion and the linear equation solution. This is easily calculated in Matlab using $\operatorname{cond}(c, 1)$ or MathCad using $\operatorname{condl}(c)$ where $c$ is the sensor matrix in question. For the ideal case the condition number of the matrix is 1 indicating a perfectly conditioned matrix. For a mathematical explanation for the procedure of calculating the condition numbers of matrices see, for example ${ }^{11,12}$ ]. Table 3 shows a comparison of condition numbers for different methods used to separate temperature and strain based on Bragg grating sensors, from which we observe that the cold hydrogenation produces a well-conditioned matrix that compares favourably with other more elaborate techniques.

Table 3. Matrix condition numbers of dual grating temperature-strain isolation configurations - a comparison. The table is ordered with the most effective method (as defined by the lowest condition number) first.

\begin{tabular}{|c|c|c|}
\hline Method & Reference & $\begin{array}{l}\text { Condition } \\
\text { Number }\end{array}$ \\
\hline Bragg gratings in different diameter fibres & James et al, [13] & 48 \\
\hline Type IIA and Type IA grating pair & Shu et al, [14] & 68 \\
\hline Superimposed grating pair & $\mathrm{Xu}$ et al, $[10]$ & 123 \\
\hline $\begin{array}{l}\text { Type I and Type IA grating pair - Cold / Hot } \\
\text { hydrogenation }\end{array}$ & Kalli et al, [This work] & $147 / 214$ \\
\hline First and second order Bragg resonances & Sivanesan et al, [11] & 161 \\
\hline $\begin{array}{l}\text { Gratings in germanosilicate and Er,Yb doped } \\
\text { germanosilicate fibres }\end{array}$ & Guan et al, [15] & 169 \\
\hline $\begin{array}{l}\text { Gratings in germanosilicate and B doped } \\
\text { germanosilicate fibre }\end{array}$ & Cavalerio et al, [16] & 173 \\
\hline Type IA and Type I grating pair & Simpson et al, [3] & 188 \\
\hline First and second order Bragg resonances & Kalli et al, $[9,17]$ & 203 \\
\hline Type IIA and Type I grating pair & Frazao et al, [18] & 272 \\
\hline \multicolumn{3}{|l|}{ Type I and IIA gratings in Ge-doped / B-Ge co-doped } \\
\hline fibres & Pal et al, [19] & $637 / 615$ \\
\hline \multicolumn{2}{|c|}{$\begin{array}{l}\text { Type-I and type-IIA gratings - no hydrogen loading, } \mathrm{Pal} \text { et al, [20] } \\
\text { high germanium doping }\end{array}$} & 750 \\
\hline
\end{tabular}




\section{CONCLUSIONS}

Work has been presented detailing the fabrication and characterisation of dual grating sensors that may be used to simultaneously decouple temperature and strain. The sensor head comprises a standard Type I grating fabricated directly adjacent to a Type IA grating, having a lower temperature coefficient and slightly higher strain coefficient. We have shown for two different fibre types that the concentration of hydrogen within the core directly affects the rate at which Type IA gratings form and the thermo-optic coefficient of the mature Type IA grating; gratings inscribed in fibre samples with a higher hydrogen concentration form faster and have lower temperature coefficients. The degree of photosensitation and hydrogen levels is controlled by the hydrogenation conditions, and typical hot and cold conditions for hydrogenation are implemented.

Using a standard matrix technique it is possible to interrogate the sensor head and decouple strain and temperature with a matrix condition number better than 150. In the case of low temperature hydrogenation there is a significant improvement in the matrix inversion errors and the condition number. This improvement is important as it augments the existing advantages of the Type1-1A dual grating sensor, namely two Bragg wavelengths having good wavelength proximity thereby avoiding costly multiplexing schemes; quick and efficient inscription using a single phase mask, common annealing cycles and the precise placement of sensors located in a common sensor head.

\section{ACKNOWLEDGEMENTS}

The authors acknowledge the UK DTI-EPSRC LINK project EMPIRE. KK and AGS respectively acknowledge Higher Technical Institute - Promotion of Research Fund and studentship of the UK EPSRC and BAE Systems.

\section{REFERENCES}

1. A.G. Simpson, K. Kalli, K. Zhou, L. Zhang and I. Bennion 2004 Formation of type IA fibre Bragg gratings in germanosilicate optical fibre Elec. Lett. 40 163-164.

2. A.G. Simpson, K. Kalli, L. Zhang, K. Zhou and I. Bennion, 2003 Abnormal photosensitivity effects and the formation of type IA FBGs BGPP, Monterey, California, MD31.

3. A.G. Simpson, K. Kalli, K. Zhou, L. Zhang and I. Bennion, 2003 An idealised method for the fabrication of temperature invariant IA-I strain sensors postdeadline session, OFS-16 Nara, Japan, PD4.

4. P. Lemaire, 1991 Reliability of optical fibres exposed to hydrogen: prediction of long-term loss increases Opt. Eng. 30 780-781.

5. J. Crank, 1975 The mathematics of diffusion Page 30. Oxford, UK: Clarendon-Press.

6. M. J. N. Lima, R. N. Nogueira, J. C. C. Silva, A. L. J. Teixeira, P. S. B. Andre, J. R. F. da Rocha, H. J. Kalinowski, and J. L. Pinto 2005 Comparison of the temperature dependence of different types of Bragg gratings Micro. Opt. Tech. Lett. 45 305-307

7. D. Jackson, 1989 Interferometers," in "Optical fibre sensors: systems and applications, Culshaw and Dakin, Eds., 2 239-280.

8. W. Jin, 1997 Simultaneous measurement of strain and temperature: Error analysis Opt. Eng., 36 598-609.

9. G. P. Brady, K. Kalli, D. J. Webb, D. A. Jackson, L. Reekie, and J. Archambault, 1997 Simultaneous measurement of strain and temperature using first- and second-order diffraction wavelengths of Bragg gratings IEE Proc. Optoelectronics, 144 156-161.

10. M. G. Xu, J. L. Archambault, L. Reekie, and J. P. Dakin, 1994 Discrimination between strain and temperature effects using dual wavelength fibre sensors Elec. Lett. 30 1085-1087.

11. P. Sivanesan, J. S. Sirkis, Y. Murata, and S. Buckley, 2002 Optimal wavelength pair selection and accuracy analysis of dual fibre grating sensors for simultaneously measuring strain and temperature Opt. Eng. 41 2456-2463, and references therein.

12. W. H. Press, B. P. Flannery, A .S. Teukolsky, and W. Vetterling, 1987 Numerical Recipes, Cambridge University Press, $2^{\text {nd }}$ Edition.

13. S. W. James, M. L. Dockney, and R. Tatam, 1996 Simultaneous independent temperature and strain measurement using in-fibre Bragg grating sensors Elec. Lett., 32 1133-1134. 
14. X. W. Shu, Y. Liu, D. H. Zhao, B. Gwandu, F. Floreani, L. Zhang, and I. Bennion, 2002 Dependence of temperature and strain coefficients on fibre grating type and its application to simultaneous temperature and strain measurement Opt. Lett. 27 701-703.

15. B. Guan, H. Tam, S. Ho, W. Chung, and X. Dong, 2000 Simultaneous strain and temperature measurement using a single fibre Bragg grating Elec. Lett. 36.

16. P. M. Cavalerio, F. M. Araujo, L. A. Ferreira, J. L. Santos, and F. Farahi, 1999 Simultaneous measurement of strain and temperature using Bragg gratings written in germanosilicate and boron-co doped germanosilicate fibres IEEE Photonics Technology Letters, 11 1635-1637.

17. K. Kalli, G. Brady, DJ. Webb, DA. Jackson, L. Zhang, and I. Bennion, 1994 Possible approach for simultaneous measurement of strain and temperature with second harmonics in a fibre Bragg grating sensor presented at OFS10, Glasgow, postdeadline paper 2.1.

18. O. Frazao, M. J. N. Lima, and J. L. Santos, 2003 Simultaneous measurement of strain and temperature using type I and type IIA fibre Bragg gratings J. Opt. A-Pure \& Appl. Opt., 5 183-185.

19. S. Pal, T. Sun, K. T. V. Grattan, S. A. Wade, S. F. Collins, G. W. Baxter, B. Dussardier, and G. Monnom, 2004 Strain-independent temperature measurement using a type-I and type-IIA optical fiber Bragg grating combination Rev. Sci. Instrum. 75 1327-1331

20. S. Pal, J. Mandal, T. Sun, K. T. V. Grattan, M. Fokine, F. Carlsson, P. Y. Fonjallaz, S. A. Wade, and S. F. Collins 2003 Characteristics of potential fibre Bragg grating sensor-based devices at elevated temperatures Meas. Sci. Techn. 14 1131-1136

*kkalli@.cytanet.com.cy; phone 35722406537; fax 35722406545 